\title{
A SHOCK-WAVE FORMULATION OF THE EINSTEIN EQUATIONS*
}

\author{
JEFFREY M. GROAH ${ }^{\dagger}$ AND BLAKE TEMPLE ${ }^{\ddagger}$
}

\begin{abstract}
We derive a weak (shock wave) formulation of the Einstein equations for a perfect fluid assuming spherical symmetry. Our purpose is to provide a framework for the weak equations that makes them amenable to mathematical methods developed for the study of shock waves in nonlinear conservation laws.
\end{abstract}

1. Introduction. We derive a weak formulation of the Einstein equations for a perfect fluid under the assumption of spherical symmetry. The weak formulation is required in order to allow for the presence of shock wave discontinuities in the fluid variables. We assume the standard gauge, and we show that in the resulting coordinates, the weak formulation of the initial value problem for the Einstein equations is valid for metrics that are only Lipschitz continuous: that is, valid for metrics that lie in the space $C^{0,1}$, the space of continuous functions with bounded difference quotients, (and hence Holder exponent one, [13]). Moreover, we show that in the standard gauge, the metric can in general be no smoother than Lipschitz continuous when shock waves are present. To clarify this, note that if $T$ is discontinuous across a smooth 3-dimensional surface $\Sigma$, then the Einstein equations $G=\kappa T$ imply that the curvature tensor $G$ will also have discontinuities across the surface. Since $G$ involves second derivatives of the metric tensor $g$, one expects that $g$ should be continuously differentiable at shock waves, with bounded second derivatives on either side, (that is, $g \in C^{1,1}$ ), in order that the equation $G=\kappa T$ will hold in the classical, pointwise a.e. sense at the shocks. However, it is known that shock-wave solutions of the Einstein equations make sense under the assumption that the metrics match only Lipschitz continuously at a shock surface, that is, $g \in C^{0,1}$. But in this case, the Lipschitz continuous matching of the metric alone is not enough to guarantee conservation at a shock, and an additional condition must be imposed to rule out the possibility that there are delta function sources in $T$ on the shock surface, $[8,15]$.

Our analysis shows that for spherically symmetric solutions of $G=\kappa T$, it is in general not possible to have metrics smoother than Lipschitz continuous, (that is, smoother than $C^{0,1}$ at shocks), when the metric is written in the standard gauge. In this paper, we show that the weak formulation is nonetheless consistent for metrics in the lower smoothness class $C^{0,1}$. This helps explain why the Oppenheimer-Snyder solution, [12], and its shock wave generalizations, $[15,16]$, involve metrics that are matched only Lipschitz continuously at an interface. Thus our results imply that when shock-waves are present, we cannot expect metrics to be smoother than these examples in the standard gauge.

The aim of this paper is to formulate the Einstein equations as a system of conservation laws with source terms. For this reason, the discussion is written to be accessible to experts in the mathematical theory of shock waves and conservation laws, $[9,5,13]$. The work here is preparatory for a subsequent paper in which the authors will give a rigorous local existence theory for shock wave solutions of these

\footnotetext{
${ }^{*}$ Received Feb. 22, 2000; revised July 17, 2000.

†Department of Mathematics, UC-Davis, Davis, CA 95616, USA (groah@math.ucdavis.edu). Supported in part by the Institute of Theoretical Dynamics (ITD), UC-Davis.

$\ddagger$ Institute of Theoretical Dynamics (Mathematical Physics) and Department of Mathematics, UC-Davis, Davis, CA 95616, USA (temple@math.ucdavi.edu). Supported in part by NSF Applied Mathematics Grant No. DMS-980-2473, and in part by the Institute of Theoretical Dynamics (ITD).
} 
equations based on a modified Glimm method, [6]. An analysis of the large time behavior of solutions to these equations would, in particular, address the issue of black-hole formation in general relativity.

2. Preliminaries. In Einstein's theory of general relativity, all properties of the gravitational field are determined by an indefinite Lorentzian metric $g$, of signature $(-1,1,1,1)$, defined on a four dimensional manifold $M$ called Spacetime. In general relativity, gravitational "forces" are identified with spacetime curvature, (as measured by the Riemann curvature tensor of the metric $g$ ), and the energy and momentum densities and their fluxes are the sources of spacetime curvature. In 1915, Albert Einstein began the subject of general relativity by introducing the Einstein gravitational field equations - the equations that describe the simultaneous evolution of the gravitational metric $g$ together with the sources. The Einstein equations can be expressed in the compact form [20],

$$
G=\kappa T \text {. }
$$

Here $G$ denotes the Einstein curvature tensor for metric $g$, and $T$ denotes the stressenergy tensor, the source of the gravitational field. Both $G$ and $T$ are symmetric tensors of rank 2. The components of the Einstein curvature tensor are given in terms of the components $R_{j k l}^{i}$ of the Riemann curvature tensor by

$$
G_{i j}=R_{i j}-\frac{1}{2} R g_{i j},
$$

where $R_{i j}$ denotes the Ricci tensor

$$
R_{i j}=R_{i \sigma j}^{\sigma},
$$

and $R$ denotes the Ricci scalar curvature

$$
R=R_{\sigma \tau}^{\sigma \tau} .
$$

The components of the Riemann curvature tensor in a given coordinate system $x$ are determined from second order derivatives of the metric tensor $g_{i j}(x)$ through the formulas

$$
R_{j k l}^{i}=\Gamma_{j l, k}^{i}-\Gamma_{j k, l}^{i}+\left\{\Gamma_{j l}^{\sigma} \Gamma_{\sigma k}^{i}-\Gamma_{j k}^{\sigma} \Gamma_{\sigma l}^{i}\right\},
$$

where the Christoffel symbols, (connection coefficients), involve first order derivatives of the metric, given by

$$
\Gamma_{j k}^{i}=\frac{1}{2} g^{\sigma i}\left\{-g_{j k, \sigma}+g_{\sigma j, k}+g_{k \sigma, j}\right\} .
$$

(We assume the Einstein summation convention whereby repeated up-down indices are to be summed from $0-3$, and indices are raised and lowered by the metric, c.f. [20].) In the case of a perfect fluid, the stress tensor takes the special form

$$
T_{i j}=(\rho+p) u_{i} u_{j}+p g_{i j},
$$

where $p$ denotes the pressure, $\rho$ the mass-energy density (as measured in the Lorentzian frame moving with the particle), and $u$ is the 4 -velocity of the fluid. The four velocity $u$, (in the tangent space $T M$ ), is the unit vector tangent to the particle path at a 
given point-that is, the components of $u$ are equal to the derivative of the coordinate parameterization of the particle path taken with respect to arclength, and hence $u$ is a unit vector relative to the spacetime metric $g$ at each point:

$$
g(u, u) \equiv g_{i j} u^{i} u^{j}=-1 .
$$

The Einstein tensor $G$ is constructed so that $\operatorname{div} G=0$ holds identically as a consequence of the Bianchi identities of geometry, [20], and thus

$$
\operatorname{div} T=0,
$$

is a consequence of the Einstein equations (2.1) alone. Here we take the covariant divergence so that it agrees with the classical divergence at the center of a locally inertial coordinate system, [15]:

$$
\begin{aligned}
\operatorname{divT} & =T_{; i}^{i j} \\
& =T_{, i}^{i j}+\Gamma_{i k}^{i} T^{k j}+\Gamma_{i k}^{j} T^{i k} .
\end{aligned}
$$

As usual, the comma denotes ordinary partial derivative, and semicolon denotes covariant derivative. It follows from (2.6) that $\Gamma_{j k}^{i}=0$ in flat Minkowski space, or to leading order in a locally inertial coordinate system, (that is, a coordinate system centered at a point where $g_{i j}=\operatorname{diag}(-1,1,1,1), g_{i j, k}=0$, for all $\left.\mathrm{i}, \mathrm{j}, \mathrm{k}\right)$. In these limits, the covariant divergence reduces to the classical divergence, and (2.9) reduces to the classical relativistic compressible Euler equations when $T$ is given by (2.7), [15]. It follows that the compressible Euler equations are a subsystem of the Einstein equations (2.1), (2.7). It is well known that the theory of the initial value problem for the compressible Euler equations is incomplete unless shock waves are incorporated into the solutions, [5, 13], and this strongly suggests that the same must be true for the Einstein equations for a perfect fluid. In this paper we derive a weak (shock wave) formulation of $(2.1),(2.7)$ that applies to spherically symmetric solutions written in the standard coordinate gauge.

A spacetime metric $g$ is said to be spherically symmetric if it takes the general form, $[20,19,7,11]$,

$$
d s^{2}=g_{i j} d x^{i} d x^{j} \equiv-A(r, t) d t^{2}+B(r, t) d r^{2}+2 D(r, t) d t d r+C(r, t) d \Omega^{2} .
$$

Here the components $A, B, C$ and $D$ of the metric are assumed to be functions of the radial and time coordinates $r$ and $t$ alone, $d \Omega^{2} \equiv d \theta^{2}+\sin ^{2}(\theta) d \phi^{2}$ denotes the line element on the 2-sphere, and $\mathbf{x} \equiv\left(x^{0}, \ldots, x^{3}\right) \equiv(t, r, \theta, \phi)$, denotes the underlying coordinate system on spacetime. (To keep track of units, we put factors of $c$ in, but to reduce the proliferation of symbols, we also use the convention $t \equiv x^{0}$ instead of the usual $c t=x^{0}$. This can be interpreted as $c=1$.) In this case we assume that the 4 -velocity $u$ is radial, by which we mean that the $x$-components of $u$ are given by

$$
u^{i}=\left(u^{0}(r, t), u^{1}(r, t), 0,0\right), \quad i=0, \ldots, 3, \text { respectively, }
$$

for some functions $u^{0}$ and $u^{1}$.

Now it is well known that in general there exists a coordinate transformation $(r, t) \rightarrow(\bar{r}, \bar{t})$ that takes an arbitrary metric of form (2.11) over to one of form, c.f., $[20]$

$$
d s^{2}=g_{i j} d x^{i} d x^{j} \equiv-A(r, t) d t^{2}+B(r, t) d r^{2}+r^{2} d \Omega^{2} .
$$


A metric of form (2.13) is said to be in the standard coordinate gauge, and it is our purpose here to establish the weak formulation of the Einstein equations for metrics of the form (2.13) in the case when $A$ and $B$ are finite, and satisfy $A B \neq 0$. It follows from our formulation that the Einstein equations together with the assumption (2.13) imply that the metric components $A$ and $B$ are only Lipschitz continuous at shock waves, that is, $A$ and $B$ are one degree less smooth than the general theory suggests they should be.

The general problem of making sense of gravitational metrics that are only Lipschitz continuous at shock surfaces was taken up in [16]. The analysis there identifies conditions that must be placed on the metric in order to insure that conservation holds at the shock, and that there do not exist delta-function sources at the shock, [8]. When these conditions are met, the methods in [16] imply the existence of a $C^{1,1}$ coordinate transformation that improves the level of smoothness of the metric components from $C^{0,1}$ up to $C^{1,1}$ at the shock. However, the results in [16] apply only to smooth interfaces that define a single shock surface for which $G=\kappa T$ holds identically on either side. For general shock wave solutions of the form (2.13), (that can contain multiplicities of interacting shock waves), it is an open question whether there exists a coordinate transformation, (say to a metric in the more general class (2.11)), that can increase the level of smoothness of the metric components by one order. For this reason, we now show that the mapping $(r, t) \rightarrow(\bar{r}, \bar{t})$ that takes an arbitrary metric of form (2.11) over to one of form (2.13), implies a loss of one order of differentiability in the metric components when shock waves are present. This argues that our results are consistent with the existence of such a smoothing coordinate transformation, but still leaves open the problem of the existence of such a transformation.

Thus we now review the construction of the mapping $(r, t) \rightarrow(\bar{r}, \bar{t})$ that takes an arbitrary metric of form (2.11) over to one of form (2.13), c.f., [20]. To start, one must assume that the metric component $C(t, r)$ in (2.11) satisfies the condition that for each fixed $t, C$ increases from zero to infinity as $r$ increases from zero to infinity, and that

$$
\frac{\partial}{\partial r} C(r, t) \neq 0 .
$$

(These are not unreasonable assumptions considering that $C$ measures the areas of the spheres of symmetry.) Define

$$
\bar{r}=\sqrt{C(r, t)} .
$$

Then the determinant of the Jacobian of the mapping $(r, t) \rightarrow(\bar{r}, t)$ satisfies

$$
\left|\frac{\partial \bar{r}}{\partial r}\right|=\frac{\partial}{\partial r} \sqrt{C(r, t)} \neq 0
$$

in light of (2.14). Thus the transformation to $(\bar{r}, t)$ coordinates is a nonsingular transformation, and in $(\bar{r}, t)$ coordinates the metric $(2.11)$ takes the form

$$
d s^{2}=-A(r, t) d t^{2}+B(r, t) d r^{2}+2 E(r, t) d t d \bar{r}+r^{2} d \Omega^{2} .
$$

(Here we have replaced $\bar{r}$ by $r$ and $A, B$ and $E$ stand in for the transformed components.) It is easy to verify that, to eliminate the mixed term, it suffices to define the time coordinate $\bar{t}$ so that, cf. [20],

$$
d \bar{t}=\phi(r, t)\{A(r, t) d t-E(r, t) d r\} .
$$


In order for (2.17) to be exact, so that $\bar{t}$ really does define a coordinate function, the integrating factor $\phi$ must be chosen to satisfy the (linear) PDE

$$
\frac{\partial}{\partial r}\{\phi(r, t) A(r, t)\}=-\frac{\partial}{\partial t}\{\phi(r, t) E(r, t)\} .
$$

But we can solve (2.18) for $\phi(r, t)$ from initial data $\phi\left(r, t_{0}\right)$, by the method of characteristics. From this it follows that, (at least locally), we can transform metrics of form (2.11) over to metrics of form (2.13) by coordinate transformation. To globalize this procedure, we need only assume that $C_{r}(t, r) \neq 0$, and that $C$ takes values from zero to infinity at each fixed $t$. Now note that in general $\phi(r, t)$, the solution to (2.18), will have the same level of differentiability as $A(r, t)$ and $E(r, t)$; and so it follows that the components of $d t$ and $d r$ in (2.17) will have this same level of differentiability. This implies that the $\bar{t}$ transformation defined by (2.17) preserves the level of smoothness of the metric component functions. On the other hand, the $\bar{r}$ transformation in (2.15) reduces the level of differentiablility of the metric components by one order. Indeed, the level of smoothness of the transformed metric component functions are in general no smoother than the Jacobian that transforms them, and by (2.15), the Jacobian of the transformation contains the terms $C_{r}$ and $C_{t}$ which will in general be only $C^{0,1}$ when $C \in C^{1,1}$. Thus, if we presume, (motivated by [15]), that for general spherically symmetric shock wave solutions of $G=\kappa T$, that there exists a coordinate system in which the metric takes the form (2.11), and the components of $g$ in these coordinates are $C^{1,1}$ functions of these coordinates, then it follows that we cannot expect the transformed metrics of form (2.13) to be better than $C^{0,1}$, that is, Lipschitz continuous. The equations we derive below allow for metrics in the smoothness class $C^{0,1}$, but in general they do not admit solutions smoother than Lipschitz continuous. It remains an open question whether solutions to these equations can be smoothed by coordinate transformation when shock waves are present.

In Section 3 we verify the equivalence of several weak formulations of the Einstein equations that allow for shock waves, and that are valid for metrics of form (2.13), in the smoothness class $C^{0,1}$. In Section 4 , we show that these equations are weakly equivalent to a system of conservation laws with time dependent sources. In a future paper, the authors will give an existence theory for these equations with general Cauchy data of bounded variation, thereby demonstrating the consistency of the Einstein equations for weak (shock wave) solutions within the class of $C^{0,1}$ metrics.

3. The Einstein Equations for a Perfect Fluid with Spherical Symmetry. In this section we study the system of equations obtained from the Einstein equations under the assumption that the spacetime metric $g$ is spherically symmetric. So assume that the gravitational metric $g$ is of the form (2.11), and to start, assume that $T^{i j}$ is any arbitrary stress tensor. To obtain the equations for the metric components $A$ and $B$ implied by the Einstein equations (2.1), plug the ansatz (2.13) into the Einstein equations (2.1). The resulting system of equations is obtained using MAPLE:

$$
\begin{aligned}
\frac{A}{r^{2} B}\left\{r \frac{B^{\prime}}{B}+B-1\right\} & =\kappa A^{2} T^{00} \\
-\frac{B_{t}}{r B} & =\kappa A B T^{01} \\
\frac{1}{r^{2}}\left\{r \frac{A^{\prime}}{A}-(B-1)\right\} & =\kappa B^{2} T^{11}
\end{aligned}
$$




$$
-\frac{1}{r A B^{2}}\left\{B_{t t}-A^{\prime \prime}+\Phi\right\}=\frac{2 \kappa r}{B} T^{22}
$$

Here "prime" denotes partial differentiation with respect to $r$, and the quantity $\Phi$ in the last equation is given by,

$$
\begin{aligned}
\Phi=-\frac{B A_{t} B_{t}}{2 A B}-\frac{B}{2}\left(\frac{B_{t}}{B}\right)^{2}-\frac{A^{\prime}}{r}+\frac{A B^{\prime}}{r B} \\
+\frac{A}{2}\left(\frac{A^{\prime}}{A}\right)^{2}+\frac{A}{2} \frac{A^{\prime}}{A} \frac{B^{\prime}}{B} .
\end{aligned}
$$

Equations (3.1)-(3.4) represent the $(0,0),(0,1),(1,1)$ and $(2,2)$ components of $G^{i j}=$ $\kappa T^{i j}$, respectively, (as indexed by $T$ on the RHS of each equation). The (3,3) equation is a multiple of the $(2,2)$ equation, and all remaining components are identically zero. (Note that MAPLE defines the curvature tensor to be minus one times the curvature tensor defined in (2.5).) It is sometimes convenient to make the change of variable $A=e^{\delta}, B=1 /(1-2 M / r),[1]$. In these variables, the Einstein equations (3.1), (3.2), and (3.3) are equivalent to

$$
\begin{aligned}
& \frac{\partial M}{\partial r}=\frac{r^{2}}{2} A \kappa T^{00}, \\
& \frac{\partial M}{\partial t}=-\frac{r^{2}}{2} A \kappa T^{01}, \\
& \frac{\partial \delta}{\partial r}=r B \kappa T^{11}+\frac{B-1}{r} .
\end{aligned}
$$

Here $M(r, t)$ is interpreted as the total mass inside radius $r$ at time $t$, and if the energy density $T^{00} \geq 0$, then (3.5) implies that $M$ is a monotone increasing function of $r$.

We are interested in solutions of (3.1)-(3.4) in the case when shock waves are present. A shock wave in the compressible Euler equations leads to discontinuities in the fluid density, pressure and velocity, and thus in light of (2.7), it follows that a shock wave would produce a discontinuity in the stress tensor $T$ at a shock. But when $T$ is discontinuous, equations (3.1)-(3.3) above imply immediately that derivatives of the metric components $A$ and $B$ are discontinuous at shocks. Moreover, if $A$ and $B$ have discontinuous derivatives when shock waves are present, it follows that (3.4), being second order, cannot hold classically, and thus equation (3.4) must be taken in the weak sense, that is, in the sense of the theory of distributions. To get the weak formulation of (3.4), multiply through by $r A B^{2}$ to clear away the coefficient of the highest (second) order derivatives, then multiply through by a test function and integrate the highest order derivatives once by parts. It follows that if the test function is in the class $C_{0}^{1,1}$, (that is, one continuous derivative that is Lipschitz continuous, the subscript zero denoting compact support), and if the metric components $A$ and $B$ are in the class $C^{0,1}$, and $T^{i j}$ is in class $L^{\infty}$, then all terms in the integrand of the resulting integrated expression are at most discontinuous, and so all derivatives make sense in the classical pointwise a.e. sense.

In order to account for initial and boundary conditions in the weak formulation, it is standard to take the test function $\phi$ to be nonzero at $t=0$ or at the specified boundary. In this case, when we integrate by parts to obtain the weak formulation, the boundary integrals are non-vanishing, and their inclusion in the weak formulation represents the condition that the boundary values are taken on in the weak sense. Thus, for example, if the boundary is $r=r_{0} \geq 0$, we say $\phi \in C_{0}^{1,1}\left(r \geq r_{0}, t \geq 0\right)$ to 
indicate that $\phi$ can be nonzero initially and at the boundary $r=r_{0}$, thereby implicitly indicating that boundary integrals will appear in the weak formulation based on such test functions.

We presently consider various equivalent weak formulations of equations (3.1)(3.4), and we wish to include the equivalence of the weak formulation of boundary conditions in the discussion. Thus, in order to keep things as simple as possible, we now restrict to the case of weak solutions of (3.1)-(3.4) defined on the domain $r \geq r_{0} \geq 0, t \geq 0$, and we always assume that test functions $\phi$ lie in the space $\phi \in C_{0}^{1,1}\left(t \geq 0, r \geq r_{0}\right)$, so that initial and boundary values are accounted for in the weak formulation. (This is the simplest case in which to rigorously demonstrate the equivalence of several weak formulations of initial boundary value problems. More general domains can be handled in a similar manner.)

Note that because (3.1)-(3.3) involve only first derivatives of $A$ and $B$, and $A, B \in$ $C^{0,1}$, it follows that (3.1)-(3.3) can be taken in the strong sense, that is, derivatives can be taken in the pointwise a.e. sense. The continuity of $A$ and $B$ imply also that the initial and boundary values are taken on strongly in any $C^{0,1}$ weak solution of (3.1) - (3.3). On the other hand, equation (3.4) involves second derivatives, and so this last equation is the only one that requires a weak formulation. The weak formulation of (3.4) is thus obtained on domain $t \geq 0, r \geq r_{0} \geq 0$ by multiplying through by a test function $\phi \in C_{0}^{1,1}\left(r \geq r_{0}, t \geq 0\right)$ and integrating by parts. This yields the following weak formulation of (3.4):

$$
\begin{aligned}
0= & \int_{r_{0}}^{\infty} \int_{0}^{\infty}\left\{-\frac{B_{t} \phi_{t}}{r A B^{2}}-\frac{B_{t} \phi}{r}\left(-\frac{A_{t}}{A^{2} B^{2}}-\frac{2 B_{t}}{A B^{3}}\right)+\frac{A^{\prime} \phi^{\prime}}{r A B^{2}}\right. \\
& \left.+A^{\prime} \phi\left(-\frac{1}{r^{2} A B^{2}}-\frac{A^{\prime}}{r A^{2} B^{2}}-\frac{2 B^{\prime}}{r A B^{3}}\right)+\frac{\phi}{r A B^{2}} \Phi+\frac{2 \kappa r}{B} \phi T^{22}\right\} d r d t \\
& -\int_{r_{0}}^{\infty} \frac{B_{t}(r, 0) \phi(r, 0)}{r A(r, 0) B^{2}(r, 0)} d r+\int_{0}^{\infty} \frac{A^{\prime}\left(r_{0}, t\right) \phi\left(r_{0}, t\right)}{r_{0} A\left(r_{0}, t\right) B^{2}\left(r_{0}, t\right)} d t
\end{aligned}
$$

Our first proposition states that the weak formulation (3.8) of equation (3.4) may be replaced by the weak formulation of the conservation laws $\operatorname{div} T=0$, so long as $A$ and $B$ are in $C^{0,1}$ and $T^{i j} \in L^{\infty}$.

Proposition 3.1. Assume that $A, B \in C^{0,1}\left(r \geq r_{0}, t \geq 0\right), T^{i j} \in L^{\infty}\left(r \geq r_{0}, t \geq\right.$ 0 ), and assume that $A, B$ and $T$ solve (3.1)-(3.3) strongly. Then $A, B$ and $T$ solve $T_{: i}^{1 i}=0$, (the 1-component of divT $=0$ ), weakly if and only if $A, B$ and $T$ satisfy (3.8).

Proof. The proof strategy is to modify (3.8) and the weak form of conservation using (3.1)-(3.3) as identities, and then observe that the two are identical at an intermediate stage. To begin, substitute for $B_{t}$ and $A^{\prime}$ in several places in (3.8) to obtain the equivalent condition

$$
\begin{aligned}
(3.9)= & \int_{r_{0}}^{\infty} \int_{0}^{\infty}\left\{\kappa T^{01} \varphi_{t}+\kappa T^{11} \varphi^{\prime}+\frac{\partial}{\partial r}\left(\varphi \frac{(B-1)}{r^{2} B^{2}}\right)+\varphi\left[-\frac{\partial}{\partial r}\left(\frac{B-1}{r^{2} B^{2}}\right)\right.\right. \\
& +\frac{B_{t}}{r}\left(\frac{A_{t}}{A^{2} B^{2}}+\frac{2 B_{t}}{A B^{3}}\right)+A^{\prime}\left(-\frac{1}{r^{2} A B^{2}}-\frac{A^{\prime}}{r A^{2} B^{2}}-\frac{2 B^{\prime}}{r A B^{3}}\right) \\
& \left.\left.+\frac{1}{r A B^{2}} \Phi+\frac{2 \kappa r}{B} T^{22}\right]\right\} d r d t \\
& +\kappa \int_{r_{0}}^{\infty} T^{01}(r, 0) \varphi(r, 0) d r+\kappa \int_{0}^{\infty} \varphi\left(r_{0}, t\right)\left[T^{11}\left(r_{0}, t\right) \frac{B\left(r_{0}, t\right)-1}{r_{0}^{2} B^{2}\left(r_{0}, t\right)}\right] d t
\end{aligned}
$$




$$
\begin{aligned}
& =\int_{r_{0}}^{\infty} \int_{0}^{\infty}\left\{\kappa T^{01} \varphi_{t}+\kappa T^{11} \varphi^{\prime}+\varphi\left[\frac{B^{\prime}(B-2)}{r^{2} B^{3}}+2 \frac{(B-1)}{r^{3} B^{2}}\right.\right. \\
& +\frac{B_{t}}{r}\left(\frac{A_{t}}{A^{2} B^{2}}+\frac{2 B_{t}}{A B^{3}}\right)+A^{\prime}\left(-\frac{1}{r^{2} A B^{2}}-\frac{A^{\prime}}{r A^{2} B^{2}}-\frac{2 B^{\prime}}{r A B^{3}}\right) \\
& \left.\left.+\frac{1}{r A B^{2}} \Phi+\frac{2 \kappa r}{B} T^{22}\right]\right\} d r d t \\
& +\kappa \int_{r_{0}}^{\infty} T^{01}(r, 0) \varphi(x, 0) d r+\kappa \int_{0}^{\infty} \varphi\left(r_{0}, t\right) T^{11}\left(r_{0}, t\right) d t .
\end{aligned}
$$

Now, the weak form of conservation of energy-momentum is given by

$$
\begin{aligned}
0= & \int_{r_{0}}^{\infty} \int_{0}^{\infty}\left\{T^{01} \varphi_{t}+T^{11} \varphi^{\prime}-\left(\Gamma_{i 0}^{i} T^{01}+\Gamma_{i 1}^{i} T^{11}\right.\right. \\
& \left.\left.+\Gamma_{00}^{1} T^{00}+2 \Gamma_{01}^{1} T^{01}+\Gamma_{11}^{1} T^{11}+2 \Gamma_{22}^{1} T^{22}\right) \varphi\right\} d r d t \\
& +\int_{r_{0}}^{\infty} T^{01}(r, 0) \varphi(x, 0) d r+\int_{0}^{\infty} \varphi\left(r_{0}, t\right) T^{11}\left(r_{0}, t\right) d t
\end{aligned}
$$

Here, we have used the fact that $T^{22}=\sin ^{2} \theta T^{33}, T^{i j}=0$ if $i \neq j=2$ or 3 , and $\Gamma_{33}^{1}=\sin ^{2} \theta \Gamma_{22}^{1}$. Next, we calculate the connection coefficients $\Gamma_{j k}^{i}$ using (2.6) to obtain,

$$
\begin{array}{ll}
\Gamma_{i 0}^{i}=\frac{1}{2}\left(\frac{A_{t}}{A}+\frac{B_{t}}{B}\right) & \Gamma_{i 1}^{i}=\frac{1}{2}\left(\frac{A^{\prime}}{A}+\frac{B^{\prime}}{B}+\frac{4}{r}\right) \\
\Gamma_{00}^{0}=\frac{A_{t}}{2 A} & \Gamma_{01}^{0}=\frac{A^{\prime}}{2 A} \\
\Gamma_{11}^{0}=\frac{B_{t}}{2 A} & \Gamma_{22}^{0}=0=\Gamma_{33}^{0} \\
\Gamma_{00}^{1}=\frac{A^{\prime}}{2 B} & \Gamma_{01}^{1}=\frac{B_{t}}{2 B} \\
\Gamma_{11}^{1}=\frac{B^{\prime}}{2 B} & \Gamma_{22}^{1}=-\frac{r}{B} \\
\Gamma_{33}^{1}=-\frac{r \sin ^{2} \theta}{B} . &
\end{array}
$$

Substituting the above formulas for $\Gamma_{j k}^{i}$ into (3.10) and using (3.1)-(3.3) as identities to eliminate some of the $T^{i j}$ in favor of expressions involving $A, B$ and $r$, we see that (3.10) is equivalent to:

$$
\begin{aligned}
0=\int_{r_{0}}^{\infty} \int_{0}^{\infty}\left\{T^{01} \varphi_{t}\right. & +T^{11} \varphi^{\prime}+\frac{\varphi}{\kappa}\left[\frac{1}{2}\left(\frac{A_{t}}{A}+\frac{3 B_{t}}{B}\right) \frac{B_{t}}{r A B^{2}}\right. \\
-\frac{1}{2}\left(\frac{A^{\prime}}{A}+\right. & \left.\frac{2 B^{\prime}}{B}+\frac{4}{r}\right) \frac{1}{r^{2} B^{2}}\left(r \frac{A^{\prime}}{A}-(B-1)\right) \\
& \left.\left.-\frac{A^{\prime}}{2 r^{2} A B}\left(r \frac{B^{\prime}}{B}+(B-1)\right)+2 \kappa \frac{r}{B} T^{22}\right]\right\} d r d t \\
& +\int_{r_{0}}^{\infty} T^{01}(r, 0) \varphi(r, 0) d r+\int_{0}^{\infty} \varphi\left(r_{0}, t\right) T^{11}\left(r_{0}, t\right) d t .
\end{aligned}
$$

After some simplification, it is clear that (3.9) is equal to (3.12). This completes the proof of Proposition 3.1.

We next show that the Einstein equations (3.1)-(3.3) together with $\operatorname{div} T=0$ are overdetermined. Indeed, we show that for weak solutions with Lipschitz continuous metric, either (3.1) or (3.2) may be dropped in the sense that the dropped equation will reduce to an identity on any solution of the remaining equations, so long as the 
dropped equation is satisfied by either the initial or boundary data, as appropriate. The following proposition addresses the first case, namely, for weak solutions in which the metric is Lipschitz continuous, the first Einstein equation (3.1) reduces to an identity on solutions of (3.2)-(3.3), so long as (3.1) is satisfied by the intial data.

Theorem 3.2. Assume that $A, B \in C^{0,1}$ and $T \in L^{\infty}$ solve (3.2), (3.3) strongly, and solve divT $=0$ weakly. Then if $A, B$, and $T$ satisfy (3.1) at $t=0$, then $A, B$, and $T$ also solve (3.1) for all $t>0$.

Proof. We first give the proof for the case when $A, B$ and $T$ are assumed to be classical smooth solutions of (3.2), (3.3) and $\operatorname{div} T=0$. This is followed by several lemmas necessary for the extension of this to the weak formulation, which is given in the final proposition. So to start, assume that $A, B$, and $T$ are all smooth functions, and thus solve $\operatorname{div} T=0$ strongly. For the proof in this case, define

$$
H^{i j} \equiv G^{i j}-\kappa T^{i j}
$$

Because (3.2) and (3.3) hold, $H^{01} \equiv H^{11} \equiv 0$. Since by assumption $T_{; i}^{i j}=0$ and since $G_{; i}^{i j}=0$ for any metric tensor as a consequence of the Bianchi identities, it follows that

$$
0=H_{; i}^{i j}=H_{, i}^{i j}+\Gamma_{i k}^{i} H^{k j}+\Gamma_{i k}^{j} H^{i k}
$$

In particular, setting $j=0$,

$$
0=H_{; i}^{i 0}=H_{, i}^{i 0}+\Gamma_{i k}^{i} H^{k 0}+\Gamma_{i k}^{0} H^{i k} .
$$

By hypothesis, $H^{i 0}=0$ when $i \neq 0$. In addition, the connection coefficients $\Gamma_{i k}^{0}$ are zero unless $i$ or $k$ equal 0 or 1 . Therefore, (3.15) reduces to the linear ODE

$$
0=H_{, 0}^{00}+\left(\Gamma_{i 0}^{i}+\Gamma_{00}^{0}\right) H^{00},
$$

at each fixed $r$. By hypothesis, $H^{00}$ is initially zero, and since we assume that $H^{00}$ is a smooth solution of (3.16), it follows that $H^{00}$ must continue to be zero for all $t>0$.

Next, assume only that $A, B \in C^{0,1}$ and $T \in L^{\infty}$ so that (3.2), (3.3) hold strongly, (that is, in a pointwise a.e. sense), but that $\operatorname{div} T=0$ is only known to hold weakly. In this case, the argument above has a problem because when $g \in C^{0,1}$, the Einstein tensor $G$, viewed as a second order operator on the metric components $A$ and $B$, can only be defined weakly when $A$ and $B$ are only Lipschitz continuous. It follows that the Bianchi identities, and hence the identity $\operatorname{div} G=0$, (which involves first order derivatives of the components of the curvature tensor), need no longer be valid even in a weak sense. Indeed, $G$ can have delta function sources at an interface at which the metric is only Lipschitz continuous, c.f. [15]. However, the above argument only involves the 0'th component of $\operatorname{div} G=0$, and the 0'th component of $\operatorname{div} G=$ 0 involves only derivatives of the components $G^{i 0}$, and as observed in (3.1), (3.2), these components only involve first derivatives of $A$ and $B$. Specifically, the weak formulation of $G_{; i}^{0 i}=0$ is given by,

$$
\begin{aligned}
0 & =\int_{r_{0}}^{\infty} \int_{0}^{\infty}\left\{-\phi_{i} G^{i 0}+\phi\left(\Gamma_{i k}^{i} G^{k 0}+\Gamma_{i k}^{0} G^{i k}\right)\right\} d r d t \\
& -\int_{r_{0}}^{\infty} \phi(r, 0) G^{00}(r, 0) d r-\int_{0}^{\infty} \phi\left(r_{0}, t\right) G^{10}\left(r_{0}, t\right) d t
\end{aligned}
$$


and since, by (3.1), (3.2), $G^{i 0}$ involves only first order derivatives of $A$ and $B$, it follows that the integrand in (3.17) is a classical function defined pointwise a.e. when $A, B \in C^{0,1}$. But (3.17) is identically zero for all smooth $A$ and $B$ because $\operatorname{div} G=0$ is an identity. Thus, when $A, B \in C^{0,1}$, we can take a sequence of smooth functions $A_{\epsilon}, B_{\epsilon}$ that converge to $A$ and $B$ in the limit $\epsilon \rightarrow 0$, (c.f. Theorem 3.4 below), such that the derivatives converge a.e. to the derivatives of $A$ and $B$. It follows that we can take the limit $\epsilon \rightarrow 0$ (3.17) and conclude that (3.17) continues to hold under this limit. Putting this together with the fact that $\operatorname{div} T=0$ is assumed to hold weakly, we conclude that

$$
H_{; i}^{0 i}=\left(G^{0 i}-T^{0 i}\right)_{; i}=0,
$$

in the weak sense, which means that $H^{00}$ is in $L^{\infty}$ and satisfies the condition

$$
\begin{gathered}
0=\int_{r_{0}}^{\infty} \int_{0}^{\infty}\left\{-\phi_{0} H^{00}+\phi\left(\Gamma_{i 0}^{i}+\Gamma_{00}^{0}\right) H^{00}\right\} d r d t \\
-\int_{r_{0}}^{\infty} \phi(r, 0) G^{00}(r, 0) d r-\int_{r_{0}}^{\infty} \phi(r, 0) H^{00}\left(r_{0}, t\right) d r .
\end{gathered}
$$

Therefore, to complete the proof of Theorem 3.2, we need only show that if $A, B$ and $T$ solve (3.2), (3.3) classically and $\operatorname{div} T=0$ weakly, then a weak $L^{\infty}$ solution $H^{00}$, (i.e., that satisfies (3.18)), of (3.16) must be zero almost everywhere if it is zero initially. Thus it suffices to prove the following proposition:

Proposition 3.3. Assume that $H, f \in L_{\text {loc }}^{\infty}(\mathbf{R} \times \mathbf{R})$. Then every $L_{l o c}^{\infty}$ weak solution to the initial value problem

$$
\begin{gathered}
H_{t}+f H=0 \\
H(x, 0)=H_{0}(x) .
\end{gathered}
$$

with initial data $H_{0} \equiv 0$ is unique, and identically equal to zero a.e., for all $t>0$.

Proof. We use the following standard theorem, [4],

THEOREM 3.4. Let $U$ be any open subset of $\mathbf{R}^{n}$. Then $u \in W_{\text {loc }}^{1, \infty}(U)$ if and only if $u$ is locally Lipschitz continuous in $U$, in which case the weak derivative of $u$ agrees with the classical pointwise a.e derivative as a function in $L_{l o c}^{\infty}(U)$.

Corollary 3.5. Let $u$ and $f$ be real valued functions, $u, f: \mathbf{R} \rightarrow \mathbf{R}$, such that $u, f \in L^{\infty}[0, T]$, and $u$ is a weak solution of the initial value problem

$$
\begin{gathered}
u_{t}+f u=0, \\
u(0)=0,
\end{gathered}
$$

on the interval $[0, T]$. Then $u(t)=0$ for all $t \in[0, T]$.

Proof of Corollary. Statement (3.20) says that the distributional derivative $u_{t}$ agrees with the $L^{\infty}$ function $f u$ on the interval $[0, T]$, and thus we know that $u \in$ $W_{l o c}^{1, \infty}(0, t)$. Therefore, by Theorem 3.4, $u$ is locally Lipschitz continuous on $(0, T)$, and the weak derivative $u_{t}$ agrees with the pointwise a.e. derivative of $u$ on $(0, T)$. Thus it follows from (3.20) that on any sub-interval $[a, b]$ of $[0, T]$ on which $u \neq 0$, we must have

$$
\frac{d}{d t}[\ln u]=\frac{u_{t}}{u}=-f, \quad \text { a.e. }
$$


Moreover, since $u$ is Lipschitz continuous, both $u$ and $\ln (u)$ are absolutely continuous on $[a, b]$, so we can integrate (3.21) to see that

$$
u(t)=u(a) e^{-\int_{0}^{t} f(\xi) d \xi},
$$

for all $t \in[a, b]$. But $u$ is continuous, so (3.22) applies in the limit that $a$ decreases to the first value of $t=t_{0}$ at which $u\left(t_{0}\right)=0$. Thus (3.22) implies that $u(t)=0$ throughout $[a, b]$, and hence we must have $u(t)=0$ for all $t \in[0, T]$, and the Corollary is true.

The proof of Proposition 3.3 now follows because it is easy to show that if $H$ is an $L^{\infty}$ weak solution of (3.19), then $H(x, \cdot)$ is a weak solution of the scalar ODE $H_{t}+f H=0$ for almost every $x$. (Just factor the test functions into products of the form $\phi_{1}(t) \phi_{2}(x)$.)

Using Proposition 3.3, we see that if equation (3.1) holds on the initial data for a solution of (3.2), (3.3), and $\operatorname{div} T=0$, then equation (3.1) will hold for all $t$. By a similar argument, it follows that if (3.2) holds for the boundary data of a solution to (3.1), (3.3), and $\operatorname{div} T=0$, then (3.2) will hold for all $r$ and $t$. We record this in the following theorem:

Theorem 3.6. Assume that $A, B \in C^{0,1}$ and $T \in L^{\infty}$ solve (3.1), (3.3) strongly, and solve divT $=0$ weakly, in $r \geq r_{0}, t \geq 0$. Then if $A, B$, and $T$ satisfy (3.2) at $r=r_{0}$, then $A, B$, and $T$ also solve (3.1) for all $r>r_{0}$.

4. The Spherically Symmetric Einstein Equations Formulated as a System of Hyperbolic Conservation Laws with Sources. Conservation of energy and momentum is expressed by the equations

$$
\begin{aligned}
0 & =(\operatorname{div} T)^{j}=T_{; i}^{i j} \\
& =T_{, i}^{i j}+\Gamma_{i k}^{i} T^{k j}+\Gamma_{i k}^{j} T^{i k},
\end{aligned}
$$

which, in the case of spherical symmetry, can be written as the system of two equations:

$$
\begin{aligned}
& 0=T_{, 0}^{00}+T_{, 1}^{01}+\Gamma_{i k}^{i} T^{k 0}+\Gamma_{i k}^{0} T^{i k} \\
& 0=T_{, 0}^{01}+T_{, 1}^{11}+\Gamma_{i k}^{i} T^{k 1}+\Gamma_{i k}^{1} T^{i k} .
\end{aligned}
$$

Substituting the expressions (3.11) for the connection coefficients (2.6) into (4.1) and (4.2), gives the equivalent system

$$
\begin{aligned}
0= & T_{, 0}^{00}+T_{, 1}^{01}+\frac{1}{2}\left(\frac{2 A_{t}}{A}+\frac{B_{t}}{B}\right) T^{00}+\frac{1}{2}\left(\frac{3 A^{\prime}}{A}+\frac{B^{\prime}}{B}+\frac{4}{r}\right) T^{01} \\
& +\frac{B_{t}}{2 A} T^{11} \\
0= & T_{, 0}^{01}+T_{, 1}^{11}+\frac{1}{2}\left(\frac{A_{t}}{A}+\frac{3 B_{t}}{B}\right) T^{01}+\frac{1}{2}\left(\frac{A^{\prime}}{A}+\frac{2 B^{\prime}}{B}+\frac{4}{r}\right) T^{11} \\
& +\frac{A^{\prime}}{2 B} T^{00}-2 \frac{r}{B} T^{22} .
\end{aligned}
$$

Now if one could use equations to eliminate the derivative terms $A_{t}, A^{\prime}, B_{t}$ and $B^{\prime}$ in (4.3) and (4.4) in favor of of expressions involving the undifferentiated unknowns $A, B$ 
and $T$, then system (4.3), (4.4) would take the form of a system of conservation laws with source terms. Indeed, $T^{00}$ and $T^{01}$ serve as the conserved quantities, $T^{10}$ and $T^{11}$ are the fluxes, and what is left, written as a function of the undifferentiated variables $\left(A, B, T^{00}, T^{01}\right)$, would play the role of a source term. (For example, in a fractional step scheme designed to simulate the initial value problem, the variables $A$ and $B$ could be "updated" to time $t_{j}+\Delta t$ by the supplemental equations (3.1) and (3.3) or (3.2) and (3.3) after the conservation law step is implemented using the known values of $A$ and $B$ at time $t_{j}$. The authors will carry this out in detail in a subsequent paper.) The system then closes once one writes $T^{11}$ as a function of $\left(A, B, T^{00}, T^{01}\right)$. There is a problem here, however. Equations (3.1)-(3.3) can be used to eliminate the terms $A_{r}, B_{t}$ and $B_{r}$, but (4.3) and (4.4) also contain terms involving $A_{t}$, a quantity that is not given in the initial data and is not directly evolved by equations (3.1)-(3.3). The way to resolve this is to incorporate the $A_{t}$ term into the conserved quantities. For general equations involving $A_{t}$, this is not possible. A natural change of $T$ variables that eliminates the $A_{t}$ terms from (4.3), (4.4), is to write the equations in terms of the values that $T$ takes in flat Minkowski space. That is, define

$$
\begin{aligned}
& T^{00}=A T_{M}^{00}, \\
& T^{01}=\sqrt{A B} T_{M}^{01}, \\
& T^{11}=B T_{M}^{11},
\end{aligned}
$$

where the subscript denotes Minkowski. In the case of a perfect fluid, $T_{M}$ takes the form

$$
\begin{aligned}
T_{M}^{00} & =\left\{\left(p+\rho c^{2}\right) \frac{c^{2}}{c^{2}-v^{2}}-p\right\}, \\
T_{M}^{01} & =\left(p+\rho c^{2}\right) \frac{c v}{c^{2}-v^{2}}, \\
T_{M}^{11} & =\left\{\left(p+\rho c^{2}\right) \frac{v^{2}}{c^{2}-v^{2}}+p\right\},
\end{aligned}
$$

where $v$ denotes the fluid speed as measured by an inertial observer fixed with respect to the radial coordinate $r$. (We discuss (4.6) in more detail in the last section.) Substituting (4.5) into (4.3), (4.4), the $A_{t}$ terms cancel out, and we obtain the system

$$
\begin{aligned}
0= & \left\{T_{M}^{00}\right\}_{, 0}+\left\{\sqrt{\frac{A}{B}} T_{M}^{01}\right\}_{, 1}+\frac{1}{2} \frac{B_{t}}{B}\left(T_{M}^{00}+T_{M}^{11}\right) \\
& +\frac{1}{2} \sqrt{\frac{A}{B}}\left(\frac{A^{\prime}}{A}+\frac{B^{\prime}}{B}+\frac{4}{r}\right) T_{M}^{01} \\
0= & \left\{T_{M}^{01}\right\}_{, 0}+\left\{\sqrt{\frac{A}{B}} T_{M}^{11}\right\}_{, 1}+\frac{1}{2} \sqrt{\frac{A}{B}}\left\{2 \frac{B_{t}}{\sqrt{A B}} T_{M}^{01}+\left(\frac{B^{\prime}}{B}+\frac{4}{r}\right) T_{M}^{11}\right. \\
& \left.+\frac{A^{\prime}}{A} T_{M}^{00}-4 r T^{22}\right\} .
\end{aligned}
$$

The following proposition states that system (4.7), (4.8) is equivalent, (in the weak sense), to the original system $\operatorname{div} T=0$.

Proposition 4.1. If $A$ and $B$ are given Lipschitz continuous functions defined on the domain $r \geq r_{0}, t \geq 0$, then $T_{M}$ is a weak solution of (4.7) and (4.8) if and only if $T$ is a weak solution of div $T=0$ in this domain. 
Proof. For simplicity, and without loss of generality, take the weak formulation with test functions compactly supported in $r>r_{0}, t>0$, so that the boundary integrals do not appear in the weak formulations. (Managing the boundary integrals is straightforward.) The variables $T_{M}^{i j}$ solve (4.7) weakly if

$$
\begin{aligned}
& 0=\int_{r_{0}}^{\infty} \int_{0}^{\infty}\{-T^{00} \varphi_{t}-\sqrt{\frac{A}{B}} T^{01} \varphi_{r} \\
&\left.+\left[\frac{1}{2} \frac{B_{t}}{B}\left(T^{00}+T^{11}\right)+\frac{1}{2} \sqrt{\frac{A}{B}}\left(\frac{A^{\prime}}{A}+\frac{B^{\prime}}{B}+\frac{4}{r}\right) T^{01}\right] \varphi\right\} d r d t \\
&(4.9)=\int_{r_{0}}^{\infty} \int_{0}^{\infty}\left\{-T_{M}^{00} A \varphi_{t}-T_{M}^{01} A \varphi_{r}\right. \\
&\left.+\left[\frac{1}{2} \frac{B_{t}}{B}\left(A T_{M}^{00}+B T_{M}^{11}\right)+\frac{1}{2} A\left(\frac{A^{\prime}}{A}+\frac{B^{\prime}}{B}+\frac{4}{r}\right) T_{M}^{01}\right] \varphi\right\} d r d t
\end{aligned}
$$

Set $\psi=A \varphi$, whereby $A \varphi_{t}=\psi_{t}-\frac{A_{t}}{A} \psi$. Using this change of test function, (4.9) becomes

$$
\begin{aligned}
0=\int_{r_{0}}^{\infty} \int_{0}^{\infty}\left\{-T^{00} \psi_{t}+T^{00} \frac{A_{t}}{A} \psi-T^{01} \psi^{\prime}+T^{01} \frac{A^{\prime}}{A} \psi\right. \\
\left.+\left[\frac{1}{2} \frac{B_{t}}{B}\left(T^{00}+\frac{B}{A} T^{11}\right)+\frac{1}{2}\left(\frac{A^{\prime}}{A}+\frac{B^{\prime}}{B}+\frac{4}{r}\right) T^{01}\right] \psi\right\} d r d t \\
=\int_{r_{0}}^{\infty} \int_{0}^{\infty}\left\{-T^{00} \psi_{t}-T^{01} \psi^{\prime}+\left[\frac{1}{2}\left(\frac{2 A_{t}}{A}+\frac{B_{t}}{B}\right) T^{00}\right.\right. \\
\left.\left.+\frac{1}{2}\left(\frac{3 A^{\prime}}{A}+\frac{B^{\prime}}{B}+\frac{4}{r}\right) T^{01}+\frac{B_{t}}{2 A} T^{11}\right] \psi\right\} d r d t
\end{aligned}
$$

which is the weak formulation of (4.3). We deduce that $T_{M}$ solves (4.7) for every Lipschitz continuous test function $\varphi$ if and only if $T$ solves (4.10), (the weak form of $T_{: i}^{0 i}=0$ ), for all Lipschitz continuous test functions $\psi$. That weak solutions of (4.8) are weak solutions of $T_{; i}^{1 i}=0$ follows by a similar argument. $\square$

It is now possible to use equations (3.1)-(3.3) as identities to substitute for derivatives of metric components $A$ and $B$, thereby eliminating the corresponding derivatives of $A$ and $B$ from the source terms of equations (4.7), (4.8). Doing this, we obtain the following system of equations:

$$
\begin{aligned}
\left\{T_{M}^{00}\right\}_{, 0}+ & \left\{\sqrt{\frac{A}{B}} T_{M}^{01}\right\}_{, 1}=-\frac{2}{r} \sqrt{\frac{A}{B}} T_{M}^{01}, \\
\left\{T_{M}^{01}\right\}_{, 0}+ & \left\{\sqrt{\frac{A}{B}} T_{M}^{11}\right\}_{, 1}=-\frac{1}{2} \sqrt{\frac{A}{B}}\left\{\frac{4}{r} T_{M}^{11}+\frac{B-1}{r}\left(T_{M}^{00}-T_{M}^{11}\right)\right. \\
+ & \text { } \left.\kappa r B\left(T_{M}^{00} T_{M}^{11}-\left(T_{M}^{01}\right)^{2}\right)-4 r T^{22}\right\} .
\end{aligned}
$$

However, depending on the choice of equation to drop, either (3.1) or (3.2), it is not clear that if we use the dropped equation to substitute for derivatives in (4.7), (4.8), that the resulting system of equations will imply that $\operatorname{div} T=0$ continues to hold, the assumption we based the substitution on in the first place. The following theorem states that (4.11), (4.12) is equivalent to $\operatorname{div} T=0$ in the weak sense: 
THEOREM 4.2. Assume that $A, B$ are Lipschitz continuous functions, and that $T \in L^{\infty}$, on the domain $r \geq r_{0}, t \geq 0$. Assume also that (3.1) holds at $t=0$, and that (3.2) holds at $r=r_{0}$. Then $A, B, T$ are weak solutions of (3.1), (3.2), (3.3) and $\operatorname{divT}=0$ if and only if $A, B, T_{M}$ are weak solutions of either system (3.1), (3.3), (4.11), (4.12), or system (3.2), (3.3), (4.11), (4.12).

Proof. Without loss of generality, we consider the case when we drop equation (3.2), and use (3.1), (3.3) and $\operatorname{div} T=0$ to evolve the metric, and we ask whether we can take the modified system (4.11) and (4.12) in place of $\operatorname{div} T=0$. In this case, we must justify the use of (3.2) in eliminating the $B_{t}$ terms in going from $\operatorname{div} T=0$ to system (4.11) and (4.12). That is, it remains only to show that equations (3.1) and (3.3) together with system (4.11) and (4.12) imply that (3.2) holds, assuming (3.2) holds at $r=r_{0}$. (If so, then by substitution, it then follows that $\operatorname{div} T=0$ also holds.)

Note that we can almost reconstruct (4.3), the first component of $\operatorname{div} T=0$, by reverse substituting (3.1), (3.3) into (4.11). To see this, first note that we can add (3.1) and (3.3) to obtain

$$
\frac{A^{\prime}}{A}+\frac{B^{\prime}}{B}-r B \kappa\left(T_{M}^{00}+T_{M}^{11}\right)=0 .
$$

Equation (4.13) is an identity that we may add to (4.11) to obtain

$$
\begin{gathered}
0=\left\{T_{M}^{00}\right\}_{, 0}+\left\{\sqrt{\frac{A}{B}} T_{M}^{01}\right\}_{, 1}-\frac{1}{2} r \sqrt{A B} \kappa\left(T_{M}^{00}+T_{M}^{11}\right) T_{M}^{01} \\
+\frac{1}{2} \sqrt{\frac{A}{B}}\left(\frac{A^{\prime}}{A}+\frac{B^{\prime}}{B}+\frac{4}{r}\right) T_{M}^{01} .
\end{gathered}
$$

Adding and subtracting

$$
\frac{1}{2} \frac{B_{t}}{B}\left(T_{M}^{00}+T_{M}^{11}\right)
$$

to the RHS of (4.14) and using

$$
H^{01}=-\frac{B_{t}}{r B}-\sqrt{A B} \kappa T_{M}^{01},
$$

(c.f. (3.2) and (3.13)), we have

$$
\begin{aligned}
0=\left\{T_{M}^{00}\right\}_{, 0} & +\left\{\sqrt{\frac{A}{B}} T_{M}^{01}\right\}_{, 1}+\frac{1}{2} \sqrt{\frac{A}{B}}\left(\frac{A^{\prime}}{A}+\frac{B^{\prime}}{B}+\frac{4}{r}\right) T_{M}^{01} \\
& +\frac{1}{2} \frac{B_{t}}{B}\left(T_{M}^{00}+T_{M}^{11}\right)+\frac{1}{2} r\left(T_{M}^{00}+T_{M}^{11}\right) H^{01}
\end{aligned}
$$

Note that all but the last term on the RHS of (4.17) is equal to the first component of $\operatorname{div} T$, and so

$$
T_{; i}^{0 i}=-\frac{1}{2} r\left(T_{M}^{00}+T_{M}^{11}\right) H^{01} .
$$

Therefore, if $A, B$, and $T_{M}$ are solutions to (3.1), (3.3), (4.17), and (4.4), it follows that

$$
\begin{aligned}
H_{; i}^{i 0} & =G_{; i}^{i 0}-\kappa T_{; i}^{i 0} \\
& =\kappa \frac{r B^{2} T^{11}}{2} H^{01}
\end{aligned}
$$


because $G_{: i}^{i 0}=0$ is an identity. But $H^{00} \equiv 0$ holds because we assume (3.1), and hence (4.18) implies that

$$
H_{, 1}^{01}+f H^{01}=0
$$

where $f \equiv \Gamma_{i 1}^{i}+2 \Gamma_{01}^{1}-\kappa \frac{r B^{2} T^{11}}{2} \in L^{\infty}$. Since we assume that $H^{01}=0$ on the boundary $r=r_{0}$, it follows from Corollary 3.5 that $H^{01} \equiv 0$.

It remains to identify conditions under which $T_{M}^{11}$ is a function of $\left(T_{M}^{00}, T_{M}^{01}\right)$ assuming that $T$ has the form of a stress tensor for a perfect fluid, (4.6). A calculation shows that, in this case, the following simplifications occur:

$$
\begin{aligned}
T_{M}^{00}-T_{M}^{11} & =\rho c^{2}-p \\
T_{M}^{00} T_{M}^{11}-\left(T_{M}^{01}\right)^{2} & =p \rho c^{2} .
\end{aligned}
$$

Using (4.19) and (4.20) we see that only the first terms on the RHS of (4.11), (4.12) depend on $v$, and the only term that is not linear in $\rho$ and $p$ is the third term on the RHS of (4.12). We state and prove the following theorem:

Theorem 4.3. Assume that $0<p<\rho c^{2}, 0<\frac{d p}{d \rho}<c^{2}$. Then $T_{M}^{11}$ is a function of $T_{M}^{00}$ and $T_{M}^{01}$ so long as $(\rho, v)$ lie in the domain $D=\{(\rho, v): 0<\rho,|v|<c\}$.

Proof. We may write (4.19) and (4.20) in the form

$$
\begin{aligned}
T_{M}^{00}-T_{M}^{11} & =f_{1}(\rho), \\
T_{M}^{00} T_{M}^{11}-\left(T_{M}^{01}\right)^{2} & =f_{2}(\rho) .
\end{aligned}
$$

Since $\frac{d f_{1}}{d \rho}=c^{2}-p^{\prime} \geq c^{2}-\sigma^{2}>0$, it follows that the function $f_{1}$ is one-to-one with respect to $\rho$. Also, $\frac{d f_{2}}{d \rho}=p^{\prime} \rho c^{2}+p c^{2} \geq p c^{2}>0$, so the function $f_{2}$ is also one-to-one in $\rho$. Consequently, the function $h=f_{2} \cdot f_{1}^{-1}$ is one-to-one, and thus

$$
T_{M}^{00} T_{M}^{11}-\left(T_{M}^{01}\right)^{2}=h\left(T_{M}^{00}-T_{M}^{11}\right)
$$

Now introduce the linear and invertible change of variables $x=T_{M}^{00}-T_{M}^{11}, y=T_{M}^{01}, z=T_{M}^{11}$, whereby (4.23) becomes

$$
(x+z) z-y^{2}=h(x) \text {. }
$$

Equation (4.24) is quadratic in $z$, and so we may solve it directly, obtaining

$$
z=\frac{-x \pm \sqrt{x^{2}+4\left(y^{2}+h(x)\right)}}{2} .
$$

From (4.25), we conclude that for any $(x, y)$, there are two values of $z$, though only one of these will correspond to values of $\rho$ and $v$ in the domain $D$. That is, since

$$
x=T_{M}^{00}-T_{M}^{11}=\rho c^{2}-p>0,
$$

and $z=T_{M}^{11}>0$, it follows that there is at most one solution of (4.25) in the domain $D$, namely

$$
z=\frac{-x+\sqrt{x^{2}+4\left(y^{2}+h(x)\right)}}{2} .
$$


We conclude that if $(\rho, v)$ lies in the domain $D$, then for each value of $T_{M}^{00}$ and $T_{M}^{01}$, there exists precisely one value of $T_{M}^{11}$.

A calculation shows that in the case $p=\sigma^{2} \rho, \sigma=$ constant, the formula for $T_{M}^{11}$ in terms of $\left(T_{M}^{00}, T_{M}^{01}\right)$ is given by

$$
T_{M}^{11}=\frac{1+2 K}{2 K}\left\{T_{M}^{00}-\sqrt{\left(T_{M}^{00}\right)^{2}-\frac{4 K}{(1+2 K)^{2}}\left(K\left(T_{M}^{00}\right)^{2}+\left(T_{M}^{01}\right)^{2}\right)}\right\}
$$

where

$$
K=\frac{\sigma^{2} c^{2}}{\left(c^{2}-\sigma^{2}\right)^{2}} .
$$

5. Statement of the General Problem. Our results concerning the weak formulation of the Einstein equations (3.1)-(3.4) assuming spherical symmetry given in Theorem 4.2 can be summarized as follows. Assume that $A, B$ are Lipschitz continuous functions, and that $T \in L^{\infty}$, on the domain $r \geq r_{0}, t \geq 0$. Then (3.1)-(3.4) are equivalent to two different systems which take the form of a system of conservation laws with source terms. In the first case, we have shown that weak solutions of the system (3.1), (3.3) together with equations (4.7), (4.8) (for $\operatorname{div} T=0$ ), will solve (3.1)(3.4) weakly, so long as (3.2) holds at $r=r_{0}$. This reduces the Einstein equations with spherical symmetry to a system of equations of the general form

$$
\begin{gathered}
u_{t}+f(u, A, B)_{x}=h_{1}\left(u, A, B, A^{\prime}, B_{t}, B^{\prime}, x\right), \\
A_{x}=h_{2}(u, A, B, x), \\
B_{x}=h_{3}(u, A, B, x),
\end{gathered}
$$

where $u=\left(T_{M}^{00}, T_{M}^{01}\right)$ agree with the conserved quantities that appear in the conservation law $\operatorname{div}_{M}=0$ in flat Minkowski space. (Here "prime" denotes $\frac{\partial}{\partial x}$ since we are using $x$ in place of $r$.) It is then valid to use equations (3.1)-(3.3) to eliminate all derivatives of $A$ and $B$ from the RHS of system (5.1), by which we obtain the system (3.1), (3.3), (4.11), (4.12), a system that closes to make a nonlinear system of conservation laws with source terms, taking the general form

$$
\begin{gathered}
u_{t}+f(u, A, B)_{x}=h_{1}(u, A, B, x), \\
A_{x}=h_{2}(u, A, B, x), \\
B_{x}=h_{3}(u, A, B, x) .
\end{gathered}
$$

Weak solutions of (5.4) will satisfy (3.2) so long as (3.2) is satisfied on the boundary $r=r_{0}$.

In the second case, we have shown that weak solutions of the system (3.2), (3.3) together with equations (4.7), (4.8) (for $\operatorname{div} T=0$ ), will solve (3.1)-(3.4) weakly, so long as (3.1) holds at $t=0$. This reduces the Einstein equations with spherical symmetry to an alternative system of equations of the general form

$$
\begin{gathered}
u_{t}+f(u, A, B)_{x}=h_{1}\left(u, A, B, A^{\prime}, B_{t}, B^{\prime}, x\right), \\
A_{x}=h_{2}(u, A, B, x), \\
B_{t}=h_{3}(u, A, B, x) .
\end{gathered}
$$

It is then valid to use equations (3.1)-(3.3) to eliminate all derivatives of $A$ and $B$ from the RHS of system (5.7), by which we obtain the system (3.2), (3.3), (4.11), 
(4.12), a system that closes to make a nonlinear system of conservation laws with source terms, taking the general form

$$
\begin{gathered}
u_{t}+f(u, A, B)_{x}=h_{1}(u, A, B, x), \\
A_{x}=h_{2}(u, A, B, x), \\
B_{t}=h_{3}(u, A, B, x) .
\end{gathered}
$$

Weak solutions of (5.10) will satisfy (3.1) so long as (3.1) is satisfied at $t=0$.

6. Wave Speeds. In this section we conclude by calculating the wave speeds associated with system (4.11)-(4.12). Because $A$ and $B$ enter as undifferentiated source terms, it follows from (4.11)-(4.12) that for spherically symmetric flow, the only wave speeds in the problem will be the characteristic speeds for the fluid. Loosely speaking, the gravitational field is "dragged along" passively by the fluid when spherical symmetry is imposed. From this we conclude that there is no lightlike propagation, (that is, no gravity waves), in spherical symmetry, even when there is matter present. (This is the conclusion of Birkoff's theorem for the empty space equations, [20].)

The easiest way to calculate the wave speeds for the fluid is from the RankineHugoniot jump conditions in the limit as the shock strength tends to zero. To start, note that the components of the 4-velocity for a spherically symmetric fluid (2.7) are $u^{0}=\frac{d t}{d s}, u^{1}=\frac{d r}{d s}, u^{2}=u^{3}=0$. Since $-1=g(u, u)$, the components $u^{0}$ and $u^{1}$ are not independent, and in particular, $-1=-\left(u^{0}\right)^{2} A+\left(u^{1}\right)^{2} B$. We define fluid speed $v$ as the speed measured by an observer fixed in $(t, r)$ coordinates. That is, the speed is the change in distance per change in time as measured in an orthonormal frame with timelike vector parallel to $\partial_{t}$ and spacelike vector parallel to $\partial_{r}$. It follows that the speed is given by $v=x / a$, where

$$
u=a \frac{\partial_{t}}{\sqrt{-g_{00}}}+x \frac{\partial_{r}}{\sqrt{g_{11}}} .
$$

Taking the inner product of $u$ with $\partial_{t}$ and then with $\partial_{r}$, we find that $a=u^{0} \sqrt{-g_{00}}$ and $x=u^{1} \sqrt{g_{11}}$, and hence

$$
v=\frac{u^{1}}{u^{0}} \sqrt{\frac{B}{A}}
$$

whereby,

$$
\left(u^{0}\right)^{2}=\frac{1}{A\left(c^{2}-v^{2}\right)} .
$$

Using (6.2) and (6.3) in (2.7), it follows that the components of the energy-momentum tensor take the following simplified form, which is valid globally in the $(t, r)$ coordinate system:

$$
\begin{aligned}
T^{00} & =\frac{1}{A}\left\{\left(p+\rho c^{2}\right) \frac{c^{2}}{c^{2}-v^{2}}-p\right\} \\
T^{01} & =\frac{1}{\sqrt{A B}}\left(p+\rho c^{2}\right) \frac{c v}{c^{2}-v^{2}} \\
T^{11} & =\frac{1}{B}\left\{\left(p+\rho c^{2}\right) \frac{v^{2}}{c^{2}-v^{2}}+p\right\} .
\end{aligned}
$$


Note that these components are equal to the components of the stress tensor in flat Minkowski space, times factors involving $A$ and $B$ that account for the fact that the spacetime is not flat. Setting

$$
\begin{aligned}
& T_{M}^{00}=\left\{\left(p+\rho c^{2}\right) \frac{c^{2}}{c^{2}-v^{2}}-p\right\}, \\
& T_{M}^{01}=\left(p+\rho c^{2}\right) \frac{c v}{c^{2}-v^{2}}, \\
& T_{M}^{11}=\left\{\left(p+\rho c^{2}\right) \frac{v^{2}}{c^{2}-v^{2}}+p\right\},
\end{aligned}
$$

it follows that

$$
\begin{aligned}
& T^{00}=T_{M}^{00} / A, \\
& T^{01}=T_{M}^{01} / \sqrt{A B}, \\
& T^{11}=T_{M}^{11} / B .
\end{aligned}
$$

The Rankine-Hugoniot jump conditions are

$$
\begin{aligned}
& s\left[T_{M}^{00}\right]=\sqrt{\frac{A}{B}}\left[T_{M}^{01}\right], \\
& s\left[T_{M}^{01}\right]=\sqrt{\frac{A}{B}}\left[T_{M}^{11}\right] .
\end{aligned}
$$

From (6.5)-(6.6), we deduce that wave speeds for the system (4.11)-(4.12) are $\sqrt{A / B}$ times the wave speeds in the Minkowski metric case, and this holds globally throughout the $(t, r)$ coordinate system. (See [14].) Eliminating $s$ from (6.5) and (6.6), yields

$$
\left[T_{M}^{01}\right]^{2}=\left[T_{M}^{00}\right]\left[T_{M}^{11}\right] .
$$

Now take the left fluid state on a shock curve to be $\left(\rho_{l}, v_{l}\right)$, and the right fluid state to be $(\rho, v)$. For a spherically symmetric perfect fluid, (6.7) defines the right velocity $v$ as a function of the right density $\rho$. Then to obtain the fluid wave speeds, just substitute this function into (6.5), solve for $s$, and take the limit as $\rho \rightarrow \rho_{l}$. Following this procedure, (6.7) simplifies to

$$
\frac{\left(v-v_{0}\right)^{2}}{\left(c^{2}-v^{2}\right)\left(c^{2}-v_{0}^{2}\right)}=\frac{[p][\rho]}{\left(p+\rho c^{2}\right)\left(p_{0}+\rho_{0} c^{2}\right)} .
$$

Note that equation (6.8) can be written as a quadratic in $v$, and hence there are two solutions. The ' + 'solutions will yield the 2 -shocks, and the '-' the 1-shocks. Dividing both sides of (6.8) by $\left(\rho-\rho_{0}\right)^{2}$ and taking the limit as $\rho \rightarrow \rho_{0}$, we see that

$$
\frac{d p}{d \rho}=\frac{\left(p+c^{2} \rho\right)^{2}}{\left(c^{2}-v^{2}\right)^{2}}\left(\frac{d v}{d \rho}\right)^{2} .
$$

Solving (6.6) for $s$ we obtain,

$$
s=\sqrt{\frac{A}{B}} \frac{\left[\left(p+\rho c^{2}\right) \frac{v^{2}}{c^{2}-v^{2}}+p\right]}{\left[\left(p+\rho c^{2}\right) \frac{c v}{c^{2}-v^{2}}\right]},
$$


and taking the limit as $\rho \rightarrow \rho_{0}$, we obtain

$$
\begin{aligned}
\lambda_{ \pm} & =\sqrt{\frac{A}{B}} \frac{\left[\left(p^{\prime}+c^{2}\right) \frac{v^{2}}{c^{2}-v^{2}}+\left(p+\rho c^{2}\right) \frac{2 v v^{\prime}\left(c^{2}-v^{2}\right)+2 v^{3} v^{\prime}}{\left(c^{2}-v^{2}\right)^{2}}+p^{\prime}\right]}{\left[\left(p^{\prime}+c^{2}\right) \frac{c v}{c^{2}-v^{2}}+\left(p+\rho c^{2}\right) \frac{c v^{\prime}\left(c^{2}-v^{2}\right)+2 c v^{2} v^{\prime}}{\left(c^{2}-v^{2}\right)^{2}}\right]} \\
& =\sqrt{\frac{A}{B}} \frac{\left[\left(p^{\prime}+c^{2}\right) \frac{v^{2}}{c^{2}-v^{2}}+\left(p+\rho c^{2}\right) \frac{2 c^{2} v v^{\prime}}{\left(c^{2}-v^{2}\right)^{2}}+p^{\prime}\right]}{\left[\left(p^{\prime}+c^{2}\right) \frac{c v}{c^{2}-v^{2}}+\left(p+\rho c^{2}\right) \frac{c v^{\prime}\left(c^{2}+v^{2}\right)}{\left(c^{2}-v^{2}\right)^{2}}\right]}
\end{aligned}
$$

(Here the plus/minus on RHS is determined by the two possible signs of $v^{\prime}=d v / d \rho$ as allowed by (6.9).) After substituting for $d v / d \rho$ using (6.9), and simplifying, we obtain

$$
\begin{aligned}
\lambda_{ \pm} & =\sqrt{\frac{A}{B}} \frac{\left[\left(p^{\prime}+c^{2}\right) \frac{v^{2}}{c^{2}-v^{2}} \pm \frac{2 c^{2} v \sqrt{p^{\prime}}}{\left(c^{2}-v^{2}\right)}+p^{\prime}\right]}{\left[\left(p^{\prime}+c^{2}\right) \frac{c v}{c^{2}-v^{2}} \pm \frac{c\left(c^{2}+v^{2}\right) \sqrt{p^{\prime}}}{\left(c^{2}-v^{2}\right)}\right]}, \\
& =\sqrt{\frac{A}{B}} \frac{\left[\left(p^{\prime}+c^{2}\right) v^{2} \pm 2 c^{2} v \sqrt{p^{\prime}}+p^{\prime}\left(c^{2}-v^{2}\right)\right]}{\left[\left(p^{\prime}+c^{2}\right) c v \pm c\left(c^{2}+v^{2}\right) \sqrt{p^{\prime}}\right]} \\
& =c \sqrt{\frac{A}{B}} \frac{\left[v^{2} \pm 2 v \sqrt{p^{\prime}}+p^{\prime}\right]}{\left[v p^{\prime} \pm\left(c^{2}+v^{2}\right) \sqrt{p^{\prime}}+c^{2} v\right]}, \\
& =c \sqrt{\frac{A}{B}} \frac{\left[v \pm \sqrt{p^{\prime}}\right]^{2}}{\left[v \pm \sqrt{p^{\prime}}\right]\left[c^{2} \pm v \sqrt{p^{\prime}}\right]} .
\end{aligned}
$$

This gives the wave speeds as:

$$
\lambda_{ \pm}=c \sqrt{\frac{A}{B}} \frac{\sqrt{p^{\prime}} \pm v}{v \sqrt{p^{\prime}} \pm c^{2}} .
$$

(For example, the formula for $\lambda_{-}$results from choosing '-' in (6.8).) The following theorem demonstrates that the system (4.11)-(4.12) is strictly hyperbolic whenever the particles are moving at less than the speed of light:

Proposition 6.1. Assume that

$$
|v|<c
$$

so that the particle trajectory has a timelike tangent vector. Then wave speeds for the general relativistic Euler equations (4.11)-(4.12) satisfy $\lambda_{-}<\lambda_{+}$.

Proof. To determine where the wave speeds are equal, set $\lambda_{-}$equal to $\lambda_{+}$and solve for $v$ to obtain $v^{2}=c^{2}$. Next, substitute $v=0$ into $\lambda_{-}$and $\lambda_{+}$to verify that $\lambda_{-}<\lambda_{+}$when $v^{2}<c^{2} A / B$. Proposition 6.1 follows directly.

As a final comment, we note that Proposition 6.1 is true because it is true in a locally inertial coordinate system centered at any point $P$ in spacetime. Indeed, in such a coordinate system, the connection coefficients vanish at $P$, and the metric components match those of the Minkowski metric to first order in a neighborhood of $P$. As a result, the general relativistic Euler equations reduce to the classical relativistic Euler equations at $P$. Since it is known in special relativity that the Euler equations are 
strictly hyperbolic for timelike particles, [14], it follows that the same must be true in general relativity. Other pointwise properties, such as genuine nonlinearity and the Lax entropy inequalities, $[13,10]$, can be verified for the spherically symmetric general relativistic equations in a similar manner.

\section{REFERENCES}

[1] Piotr Bizon, Private Communication.

[2] R. Courant and K. Friedrichs, Supersonic Flow and Shock-Waves, Wiley-Interscience, 1948 \& 1972 .

[3] A. Einstein, Der feldgleichungen der gravitation, Preuss. Akad. Wiss., Berlin, Sitzber, 314 (1970), pp. 529-548.

[4] Lawrence C. Evans, Partial Differential Equations, Vol 3A, Berkeley Mathematics Lecture Notes, 1994.

[5] J. GLimm, Solutions in the large for nonlinear hyperbolic systems of equations, Comm. Pure Appl. Math., 18 (1965), pp. 697-715.

[6] J. GROAH AND B. Temple, Shock-wave solutions of the spherically symmetric Einstein equations with perfect fluid sources: existence and consistency for the intitial value problem, in preparation.

[7] S. W. Hawking and G. F. R. Ellis, The Large Scale Structure of Spacetime, Cambridge University Press, 1973.

[8] W. IsRAeL, Singular hypersurfaces and thin shells in general relativity, IL Nuovo Cimento, Vol. XLIV B, N. 1 (1966), pp. 1-14.

[9] P. D. LAx, Hyperbolic systems of conservation laws, II, Comm. Pure Appl. Math., 10 (1957), pp. $537-566$.

[10] P. D. Lax, Shock-waves and entropy, in Contributions to Nonlinear Functional Analysis, E. Zarantonello, ed., Academic Press, 1971, pp. 603-634.

[11] C. Misner, K. Thorne, and J. Wheeler, Gravitation, Freeman, 1973.

[12] J. R. Oppenheimer and J. R. Snyder, On continued gravitational contraction, Phys. Rev., 56 (1939), pp. 455-459.

[13] J. Smoller, Shock-Waves and Reaction-Diffusion Equations, Springer Verlag, 1983.

[14] J. Smoller And B. Temple, Global solutions of the relativistic Euler equations, Comm. Math. Phys., 157 (1993), pp. 67-99.

[15] J. Smoller And B. Temple, Shock-wave solutions of the Einstein equations: the OppenheimerSnyder model of gravitational collapse extended to the case of non-zero pressure, Arch. Rat. Mech. Anal., 128 (1994), pp. 249-297.

[16] J. Smoller And B. Temple, Astrophysical shock-wave solutions of the Einstein equations, Phys. Rev. D, 51:6 (1995), pp. 2733-2743.

[17] J. Smoller And B. Temple, Shock-wave solutions in closed form and the Oppenheimer-Snyder limit in general relativity, SIAM J. Appl. Math, 58:1 (1998), pp. 15-33.

[18] J. Smoller And B. Temple, Cosmology with a shock wave, preprint.

[19] R. M. Wald, General Relativity, University of Chicago Press, 1984.

[20] S. Weinberg, Gravitation and Cosmology: Principles and Applications of the General Theory of Relativity, John Wiley \& Sons, New York, 1972. 